\title{
Pensamento Computacional nos ensinos fundamental e médio: uma revisão sistemática*
}

\author{
Adriana Bordini ${ }^{1}$, Christiano Avila ${ }^{1}$, Mônica Cunha ${ }^{1}$, \\ Simone Cavalheiro ${ }^{1}$, Luciana Foss ${ }^{1}$ \\ ${ }^{1}$ Programa de Pós-Graduação em Computação - Universidade Federal de Pelotas (UFPel) \\ 96.010-610 - Pelotas - RS - Brasil \\ \{adriana.bordini, simone.costa\}@inf.ufpel.edu.br
}

\begin{abstract}
This paper presents a systematic review with the goal of describing the state of the art on the topic Computational Thinking in the scope of primary and secondary education. We attempt to identify the main approaches that focus on the theme, describing its goals, pedagogical practices, the tools used and/or proposed, as well as the concepts and skills that have been developed.
\end{abstract}

Resumo. Este trabalho apresenta uma revisão sistemática com o objetivo de descrever o estado da arte sobre o tema Pensamento Computacional no Ensino Fundamental e Médio. Procurou-se identificar as principais abordagens neste tema, descrevendo seus objetivos, práticas pedagógicas, as ferramentas utilizadas elou propostas, bem como os conceitos e habilidades desenvolvidos.

\section{Introdução}

As inovações tecnológicas possibilitam a realização de tarefas do dia a dia de forma inimaginável até alguns anos atrás. Estas mudanças também alteram as habilidades necessárias para a resolução de problemas, tanto na vida cotidiana como na educação do século XXI. Jeannette Wing [Wing 2006] descreveu o Pensamento Computacional (PC) como um processo de resolução de problemas, fundamentado na Ciência da Computação (CC), capaz de desenvolver muitas destas habilidades (tais como: abstração, automação, análise, entre outras). Em particular, a Computer Science Teachers Association (CSTA) e a International Society for Technology in Education (ISTE) junto com colaboradores propuseram a definição operacional do PC, delineando seu escopo e dimensões.

Desde então, diversos trabalhos propuseram a introdução de conceitos de computação na educação, bem como o desenvolvimento de habilidades do PC em seus diferentes níveis. Pesquisas já relatam as diferentes estratégias adotadas para a introdução da computação na Educação Básica [Bordini et al. 2016a, Martins et al. 2016, Mota et al. 2014], bem como descrevem os desdobramentos do PC [Bordini et al. 2016b, Oliveira et al. 2016, Andrade et al. 2013], no Brasil.

Este trabalho apresenta uma revisão sistemática com o objetivo de descrever o estado da arte (mundial) sobre o tema PC no escopo do Ensino Fundamental e Médio. Neste levantamento buscou-se caracterizar os principais objetivos e metodologias dos trabalhos dos últimos 5 anos sobre o tema. O texto está organizado como segue. A seção 2 apresenta a metodologia deste levantamento. Na seção 3 as abordagens identificadas são descritas. Por fim, na seção 4, são apresentadas as considerações finais.

*Projeto com chancela da SBC e realizado com o apoio da PREC e PRPPG / UFPel. 
VI Congresso Brasileiro de Informática na Educação (CBIE 2017)

Anais do XXVIII Simpósio Brasileiro de Informática na Educação (SBIE 2017)

\section{Metodologia do Levantamento Realizado}

Este levantamento seguiu a metodologia Revisão Sistemática de Literatura (RSL). Inicialmente, identificou-se que não havia um levantamento já realizado neste escopo e definiuse o protocolo da pesquisa. O objetivo do levantamento foi o de identificar, interpretar e avaliar pesquisas relevantes na área do PC no âmbito dos Ensinos Fundamental e Médio. Considerou-se os artigos publicados nos últimos 5 anos em periódicos e anais de conferências internacionais, abrangendo o período de 2012 a agosto/2016, no idioma inglês. Optou-se por realizar a busca dos trabalhos nas principais bases de dados científicas digitais on-line: IEEE, ACM, Scopus, Science Direct e Springer. Na busca selecionou-se artigos que continham as palavras "computational thinking" no título ou no resumo ou nas palavras chaves e as palavras education ou school ou $k$ - 12 ou teach ou learn em qualquer parte do texto. Para fazer a seleção dos artigos que se enquadravam no foco da pesquisa, definiu-se os seguintes critérios de inclusão: I1 - trabalhos publicados em periódicos e conferências de Qualis A1 a B1 e I2 - trabalhos sobre PC com foco na Educação Básica. Os critérios de exclusão considerados foram: E1 - trabalhos incompletos, tais como, artigos curtos, pôsteres, painéis, apresentações; E2 - outras revisões de literatura na área; E3 - trabalhos duplicados; E4 - trabalhos publicados de um mesmo projeto, somente o mais recente foi considerado. Na sequência, efetivamente realizou-se o levantamento dos trabalhos. Aplicando a string de busca no período descrito retornaram 1192 artigos. Considerando o critério I1, resultaram em 236 artigos. Por meio de uma leitura e análise do resumo, aplicou-se os demais critérios de inclusão e exclusão, totalizando 132 artigos. Os textos dos 132 trabalhos foram lidos na íntegra, e novamente aplicou-se os mesmos critérios, resultando 80 artigos. No que segue sumariza-se o relato desta revisão.

\section{Pensamento Computacional nos Ensinos Fundamental e Médio}

A relação completa dos artigos considerados neste levantamento está na Tab. 1. Cada artigo é referenciado pelo respectivo ID descrito na Tabela. Particularmente foram identificados trabalhos que: buscaram desenvolver habilidades do PC (através da programação (PROG) (6 artigos), da robótica (ROBO) (7 artigos), de jogos (JOGO) (2 artigos), do uso e/ou criação de ferramentas (FERR) (5 artigos) ou da introdução de conceitos de computação (CONC) (5 artigos)), investigaram a inserção da computação no currículo (CURR) (10 artigos), apresentaram metodologias para o desenvolvimento do PC (METO) (9 artigos), propuseram metodologias de avaliação do PC (AVAL) (17 artigos), buscaram a integração da computação em outras disciplinas (DISC) (10 artigos), realizaram intervenções com professores da educação básica (PROF) (7 artigos) e investigaram a percepção da comunidade escolar sobre PC (PERC) (2 artigos). Trabalhos com mais de um objetivo, foi considerado na categoria de maior abrangência. A última coluna da Tab. 1 identifica o público-alvo de cada trabalho: Ensino Fundamental (EF) ou Ensino Médio (EM). Quando o trabalho não especifica público, utiliza-se a nomenclatura NE. A caracterização das abordagens foi realizada por meio de respostas às questões de pesquisa descritas a seguir.

QP1 - Quais são os objetivos, ferramentas utilizadas e/ou desenvolvidas e as práticas pedagógicas adotadas em cada uma das abordagens?

Avaliação do PC: Dos 80 artigos, 17 (ID 1 ao 17) tiveram como objetivo principal a avaliação de habilidades do PC, seja através da análise dos resultados de experiências 
VI Congresso Brasileiro de Informática na Educação (CBIE 2017)

Anais do XXVIII Simpósio Brasileiro de Informática na Educação (SBIE 2017)

de ensino, da criação ou uso de ferramentas ou da utilização de métodos já conhecidos para a criação de uma avaliação própria. Para atingirem estes objetivos, foram realizadas práticas pedagógicas utilizando ambientes lúdicos [16] e/ou de programação visual [2], propostas de ensino e aprendizagem ativa [15], o aprender fazendo [7], os desafios [1, 11] e a aprendizagem scaffolding [13] (variedade de técnicas de instrução usadas para mover os alunos progressivamente em direção a uma maior compreensão do assunto tratado).

Dentre as ferramentas utilizadas para o desenvolvimento das atividades estão os ambientes de programação visual Scratch [7,11, 15 e 17], Alice [1], AgentCubes [12] e o AgentSheets [14]. Além dos processos mais usuais de avaliação, tais como: produções dos alunos, observações, questionários de opinião, entrevistas, pré- e pós-testes; alguns trabalhos incluem outras abordagens para avaliar o desenvolvimento de habilidades. As Taxonomias Bloom [3, 7, 15] e SOLO [11] foram utilizadas para classificar as respostas dos alunos aos desafios de programação. A expansão da ferramenta Hairball [4] foi proposta para detectar erros de programação em projetos Scratch. A ferramenta Fairy Assessment [1], criada para o Alice, visa a avaliação de habilidades do PC por meio da resolução de desafios e criação de jogos. O REACT [12], sistema cyberlearning foi concebido para avaliar em tempo real dados correspondentes a cada projeto (jogo) do estudante criado no AgentCubes. A plataforma VIVA [13] propõe uma taxonomia com 15 questões (independentes) de computação, para avaliar a capacidade do aluno na resolução de problemas (com ou sem programação). Em [3], apresenta-se um quadro do PC para ser usado como uma ferramenta de planejamento e avaliação de projetos no jogo educativo Light-Bot.

PC por meio da introdução de conceitos de computação: Em 5 trabalhos (ID 18 ao 22) propôs-se o desenvolvimento de habilidades do PC através da introdução de conceitos de computação. Nesta categoria estão incluídos os trabalhos que eventualmente incluíram programação, robótica e/ou jogos, mas cujo foco foi dado ao ensino de conceitos de computação. Dentre os objetivos pretendidos, destacam-se: aumentar o interesse dos alunos pela área da CC [20], alguns em especial do gênero feminino [19]; apresentar a área da computação e esclarecer o que um profissional da área faz [20]. Estes objetivos foram trabalhados através de práticas pedagógicas de aprendizagem baseada em problemas [18] e aprendizagem scaffolding [19]. Dentre as ferramentas utilizadas, destaca-se a linguagem de programação visual Scratch [19], a placa de circuito impresso Serious Toy [20] e o jogo de tabuleiro Chainreaction [21]. Para a avaliação da prática pedagógica utilizaram entrevistas semi-estruturadas, pré- e pós-testes e questionários de opinião.

PC por meio da Programação: Seis trabalhos (ID 68 ao 73) desenvolveram habilidades do PC através da programação. Os trabalhos desta abordagem tinham objetivos variados: atrair a atenção do público em geral (alguns, em particular do feminino [70]); refletir sobre a educação de programação no K-12 [68]; abordar o processo de transição de linguagens gráficas para linguagens textuais [72]; e ensinar programação através da depuração de programas [69]. As propostas de ensino aprendizagem também foram diversas, por meio do(a): uso e/ou criação de jogos $[69,71]$, transição de linguagem visual para textual [72], compartilhamento de projetos [73], computação desplugada [72], aprender fazendo [73], proposta de desafios [69], clubes de programação baseados no modelo Bridge 21 [70], scaffolding [72, 73] e uso da versão on-line do FACT [73] (utiliza uma abordagem scaffolded e aprendizado cognitivo para modelar o processo de programação). Dentre as ferramentas utilizadas, destaca-se o Scratch [68, 70, 72, 73], jogos Gidget [69] e Blockly 
VI Congresso Brasileiro de Informática na Educação (CBIE 2017)

Anais do XXVIII Simpósio Brasileiro de Informática na Educação (SBIE 2017)

[70]. Outros trabalhos utilizaram linguagens de programação: C\# [71], Python [72] e UPL [72]. As avaliações ocorreram através de: observações, pré- e pós-questionários (de opinião), pré- e pós-testes e análise de código (produção dos alunos).

PC por meio de Jogos: Em 2 trabalhos (ID 45 e 46) a proposta de ensino-aprendizagem foi através do uso e/ou criação de jogos para promover o PC. Em [45] os alunos criaram jogos com o ambiente de programação visual Alice e em [46] utilizaram o jogo de quebra-cabeça GrACE para estimular o PC através da Procedural Content Generation [Togelius et al. 2011] (prática de criar conteúdo de jogo automaticamente) e da colaboração. Avaliaram os resultados com pré- e pós-testes e pré- e pós-questionários.

PC por meio da robótica: Com 7 trabalhos (ID 74 ao 80), a robótica foi outra abordagem frequentemente adotada para o desenvolvimento de habilidades do PC. Dentre as propostas de ensino-aprendizagem, algumas surgem com maior frequência, que é o caso da aprendizagem Scaffolding [74, 75]. Outras propostas são baseadas na metodologia de projetos [80], no trabalho com desafios [75], na proposta do aprender fazendo (mão na massa) [80], nas competições [75, 80] e na proposta lúdica com computação vestível [78].

Dentre as ferramentas utilizadas tem-se os kits de robótica Lego Mindstorms NXT e Arduino, voltados para a educação tecnológica, assim como os ambientes de programação visual Ardublock e CREATE Lab Visual Programmer. Em [75, 79, 80] o kit Lego foi utilizado com o objetivo de estimular o interesse dos estudantes para a área da computação. Através de desafios os alunos realizaram a construção básica de um robô, depois foram encarregados de equipar seus robôs com sensores e atuadores. Em [77, 80] usaram a plataforma Arduino para desenvolver a lógica de programação. Em [77] propôsse um jardim robô controlado com Python e em [78] criaram personagens lúdicos com o Arduino Lilypad (placa costurada dentro de tecidos), programados com Ardublock. O CREATE [76] foi proposto com o objetivo de facilitar a programação de comportamentos complexos e interações de robôs no projeto Arts \& Bots.

Integração da computação em outras disciplinas: Nesta abordagem, 10 artigos (ID 56 ao 65) desenvolveram habilidades do PC integrando fundamentos da computação em 3 áreas: humanas, ciências e artes. $\mathrm{Na}$ área das humanas os tópicos trabalhados foram: leitura e compreensão de texto, literatura inglesa e linguagem; na área das ciências as disciplinas foram: ciências, matemática (geometria, expressões, etc.), física (cinemática, música), química e ecologia; e na área de artes, música. As práticas adotadas foram: oficinas para trabalhar os conceitos do DISSECT (algoritmos, sequência de passos, ramificação, iteração, variáveis, clareza, correção, eficiência, abstração e o PC) com professores e alunos [57, 61 e 65]; análise de interação de discurso [59] e a colaboração através do compartilhamento na plataforma de e-learning no projeto Problem Posing \& Solving [63]. Dos objetivos, destacam-se: mostrar a utilidade e praticidade das técnicas de modelagem para professores e estudantes [64]; preencher lacunas entre PC e a área de humanas [65]; aumentar o interesse pela área da computação [60]; impulsionar o ensino e aprendizagem da matemática e informática no EM [63].

As ferramentas utilizadas foram: ToonDoo [65] que permite a criação de quadrinhos on-line; ferramentas do Google para a criação de blogs, documentos, apresentações e planilhas [65]; Scratch [56, 57, 61, 62] e ViMAP [59] (ambiente de programação visual e de modelagem baseada em agentes); linguagem Python [57]; plataforma de e-learning 
VI Congresso Brasileiro de Informática na Educação (CBIE 2017)

Anais do XXVIII Simpósio Brasileiro de Informática na Educação (SBIE 2017)

Moodle Maple Suite [63] (para trabalhar com a matemática); Minecraft [60] e o CTSiM [58] (ambiente de aprendizagem para PC e ciências). As atividades realizadas nos artigos foram avaliadas de muitas formas, por meio: de códigos produzidos; de pré- e pós-testes; do teste padrão California Standards Test e da análise de interação entre alunos.

Intervenção com professores da educação básica: Sete artigos (ID 38 ao 44) trabalharam conceitos de computação e/ou do PC com professores dos EF e EM, com o objetivo principal de incentivar a disseminação da área. As intervenções foram através de execuções de planos de aula [39, 40]; do aprender fazendo [44]; de propostas de metodologias e ferramentas para a inserção do PC na sala de aula [38, 43]; e relato das dificuldades de inserção da computação na sala de aula [38, 41, 42]. Dentre as metodologias de ensino, utilizaram: o modelo pedagógico Bridge21 [43], o projeto de jogos [43], aprendizagem baseada em problemas [44], aprendizagem ativa e cooperativa com trabalhos em duplas [44]. As ferramentas utilizadas nas oficinas foram: a ferramenta Entry [44] (aprendizagem baseada em problemas) e os ambientes de programação Scratch [39, 40-42], Alice [40] e Etoys [42]. Avaliaram através de questionários e da produção dos professores (planos de aula, códigos, etc.).

Inserção da computação no Currículo: Do total, 10 artigos (ID 23 ao 32) propuseram a inclusão da CC e/ou PC no currículo dos EF e EM. Destes, 8 propuseram a computação como disciplina (dos quais, 7 com foco em algoritmos e programação) e 2 artigos propuseram a inclusão da computação de forma interdisciplinar. Os objetivos delineados foram: promover o PC (para meninas) [26]; inserir princípios de legalidade (por exemplo, regras de utilização de uma ferramenta ou até do uso de um jogo) [30]; propor um modelo de desenvolvimento profissional com foco no Computer Science Principles [29]; trazer a CC para a Educação Básica [31]; refletir sobre as maneiras que a computação e as tecnologias são utilizadas na pesquisa e na indústria, trazendo-as para a educação tradicional [24]; trazer a computação para outras disciplinas (biologia, história, artes gráficas, inglês, latim e informática) [23]. Para realizarem estes objetivos, propuseram atividades: com e sem o uso do computador (computação desplugada [Bell et al. 1998]) [26, 27, 29, 31]; que envolvessem projetos com práticas da transição de linguagem visual para textual [32]; que incentivassem a criatividade e o aprendizado ativo [31]; que utilizassem de sites de programação [29]; que promovessem a criação e o desenvolvimento de clubes de programação, eletrônica e tecnologia de artesanato [24].

As ferramentas utilizadas/sugeridas foram: Scratch [25, 27], Alice [26, 32], Kodu [30], Logo [23] e Python [32]; artefatos de robótica educacional (Lego [26], Arduino [24], Arduino Lilypad [24], Dash [31] e Thymio [31]); sites de programação (Code.org [25, 31] e Hora do Código [29]). A avaliação foi tanto quantitativa através de testes, quanto qualitativa, através de observações, entrevistas de grupo e pré- e pós- questionários.

Metodologias de implantação: Do total, 9 artigos (ID 47 ao 55) sugeriram propostas pedagógicas para promover habilidades do PC. Três trabalhos [47, 50, 51] estavam no escopo do projeto Scalable Game Design (SGD), com diferentes objetivos: propor a criação de jogos e simulações como forma de ampliar o interesse pela área, relatar resultados do projeto Computational Thinking Acquisition no Brasil e propor a tecnologia PoliFacets (ambiente que permite explicitar conceitos do AgentSheets). Dois trabalhos $[52,54]$ propuseram atividades pedagógicas com o Alice: um através da dança, com o ob- 
VI Congresso Brasileiro de Informática na Educação (CBIE 2017)

Anais do XXVIII Simpósio Brasileiro de Informática na Educação (SBIE 2017)

jetivo de mostrar aplicações alternativas em computação e o outro pela extensão do Alice para adaptação à cultura do Oriente Médio. Nos demais trabalhos, propôs-se: um método de dimensão descendente, o Computational Thinking Model based on Blended Learning, com o objetivo de auxiliar os professores a usar computadores no processo de solução de problemas [53]; um método de ensino modular baseado em casos práticos, projetos de engenharia e características disciplinares, com o objetivo de aproximar a computação de outras disciplinas [49]; uma oficina de programação com o aplicativo App Inventor for Android onde utilizaram uma pedagogia de discurso intensivo para introduzir programação e conceitos da computação no EF [48]; e uma adaptação de atividades da computação desplugada para o contexto da sala de aula [55].

As práticas de ensino aprendizagem utilizadas para atingirem os diversos objetivos citados, foram: criação de jogos, simulações e animações [47, 50, 51]; a dança [52]; integração multidisciplinar [49]; computação desplugada [55]; desenvolvimento de planos de aula [55] e blended learning [53]. As ferramentas foram diversas: jogos Frogger [47], Pacman [47] e Space Invaders [47]; ambiente de documentação ao vivo PoliFacets [51] e ambientes de programação visual AgentSheets [47, 50, 51], Alice [52, 54] e App Inventor for Android [48]. As avaliações foram por meio de: observações das práticas dos professores; coleta de dados empíricos com gravações de áudio, vídeo e anotações; entrevistas com professores e alunos, com o uso de técnicas de análise de discurso; análise de códigos produzidos; questionários e uso de rubricas (fornecendo diretrizes de como classificar as respostas como proficiente, parcialmente proficiente ou insatisfatórias).

PC por meio de outras ferramentas: Do total de artigos analisados, 5 (ID 33 ao 37) usaram, criaram e/ou complementaram ferramentas para trabalharem habilidades do PC. Dentre as ferramentas criadas, tem-se: a Bags [36] para o ensino de álgebra relacional e análise de dados; o Simulation Creation Toolkit [34] para criar simulações predador/presa baseadas nos Computational Thinking Patterns e o Code Bits [37] um conjunto de ferramentas de PC tangível, que faz uso da tecnologia realidade aumentada em dispositivos móveis para processar código. Em [33] exploraram a ferramenta AgentSheets utilizando 3 métodos de pesquisa qualitativos, para ajudar a compreensão de processos de aprendizagem suportada pela interface. E em [35] criaram a extensão AliCe-ViLlagE do Alice para explorar a programação em pares. Nesta abordagem, as práticas de ensino envolveram a utilização de ambientes de programação visual e o uso e/ou criação de jogos.

Percepção da comunidade escolar sobre o tema PC: Dois trabalhos (ID 66 e 67) distribuíram questionários para professores com o objetivo de verificar a percepção deles sobre o tema PC. Em [66] investigaram a melhor forma de tornar a computação interessante aos alunos e em [67] vislumbraram opções de como e quando inserir o PC.

QP2 - Quais são as habilidades do PC que têm sido trabalhadas e quais são os conceitos de computação abordados? As Figuras 1(a) e 1(b) relacionam, respectivamente, as abordagens com as habilidades e conceitos desenvolvidos. A maioria dos artigos, 57,5\% se concentram nas abordagens AVAL, DISC, CURR e METO. Nas 4 abordagens, as habilidades do PC mais trabalhadas foram as mesmas: pensamento algorítmico, resolução de problemas e abstração. Os conceitos de computação mais trabalhados foram praticamente os mesmos: programação em AVAL, CURR e METO e algoritmos em AVAL, CURR e DISC. Observou-se que tanto no desenvolvimento de algoritmos quanto na programação, 
VI Congresso Brasileiro de Informática na Educação (CBIE 2017)

Anais do XXVIII Simpósio Brasileiro de Informática na Educação (SBIE 2017)

as atividades geralmente incluem os conceitos de variáveis, sequência, condicional e repetição. Percebeu-se também que a introdução de tais conceitos envolve o desenvolvimento das habilidades: abstração, resolução de problemas e pensamento algorítmico.

Conforme Figura 1(a), outras habilidades também têm sido avaliadas, tais como: colaboração, generalização, simulação e pensamento lógico. Habilidades que tiveram apenas uma ocorrência foram omitidas da Figura 1(a): pensamento estratégico [21] e raciocínio formal [18] na CONC, análise dados [36] na FERR e automação [40] na PROF.

Igualmente, conforme Figura 1(b), observa-se que diversos outros conceitos, além dos já citados, são considerados, entre eles: simulação, decomposição, modelagem, paralelismo, depuração e representação de dados. Conceitos da computação que tiveram apenas uma ocorrência foram omitidos da Figura 1(b), sendo eles: raciocínio indutivo [5] na AVAL; protocolos [20] na CONC; álgebra relacional [36] na FERR; concorrência [43] e sistemas embarcados [43] na PROF; criptografia [55] na METO; e modelo E-R [64], modularização [62] e portas lógicas [60] na DISC. Além das habilidades do PC outras foram consideradas relevantes, tais como: criatividade [2, 26, 29, 39, 44, 76 e 80], leitura [11, 64, 65], escrita [65], compreensão de texto [64] e operações algébricas [56].

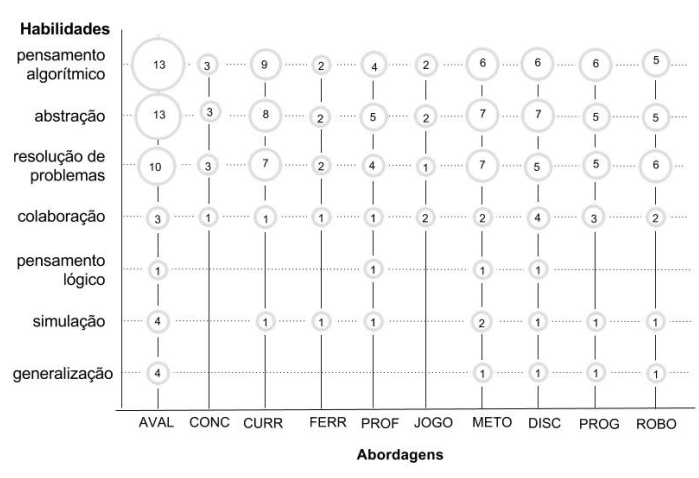

(a) Habilidades do PC por abordagem.

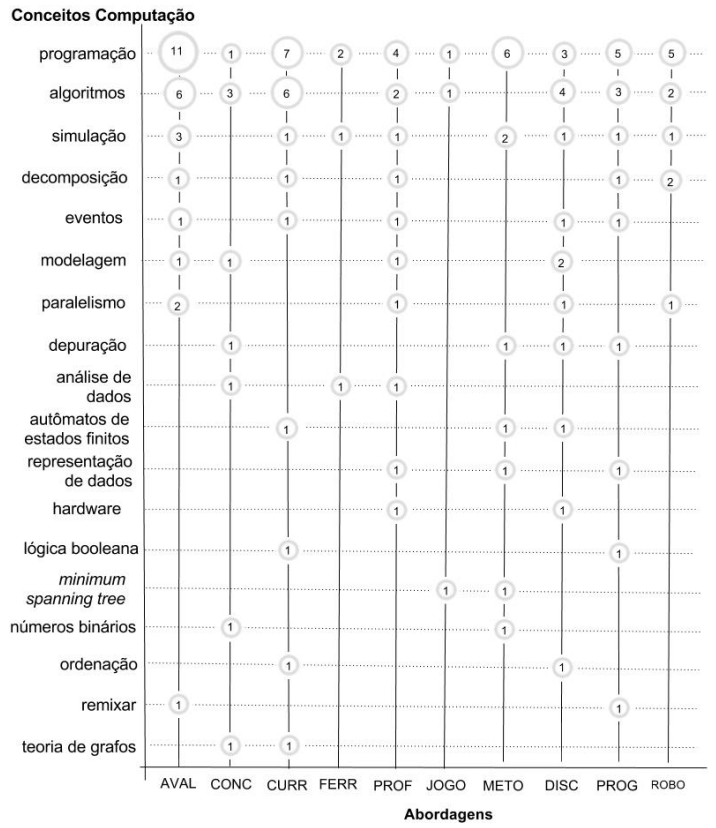

(b) Conceitos do PC por abordagem.

Figura 1. Habilidades e Conceitos do PC por abordagem.

\section{Considerações}

Este trabalho apresenta um panorama geral das principais abordagens e metodologias que se tem utilizado para a inclusão do PC nos Ensinos Fundamental e Médio. O relato e análise apresentados permitem que educadores reflitam sobre as diferentes estratégias adotadas, possivelmente adequando para a sua realidade. Neste estudo foram identificadas diferentes abordagens, predominando: "avaliação do PC", onde se destacou o uso e/ou criação de ferramentas para a avaliação de habilidades do PC; "integração da computação com outras disciplinas", onde as disciplinas da área de ciências, matemática e física, são as 
VI Congresso Brasileiro de Informática na Educação (CBIE 2017)

Anais do XXVIII Simpósio Brasileiro de Informática na Educação (SBIE 2017)

mais citadas; "inserção da computação no currículo", onde a maioria dos trabalhos propõe a computação como disciplina; "metodologias de implantação", envolvendo propostas geralmente inseridas em desenvolvimento de jogos.

Observou-se que aulas de programação são as práticas pedagógicas mais utilizadas, onde geralmente são propostas através da metodologia de projetos, o que facilita e incentiva a programação em pares e/ou colaboração. A abordagem scaffolding também aparece em muitos trabalhos auxiliando a construção do conhecimento. $\mathrm{O}$ foco tem sido nas habilidades de abstração e pensamento algorítmico, as quais são trabalhadas através dos conceitos de algoritmos e programação. Dentre as linguagens de programação que predominam, as visuais são as mais adotadas. Quanto as metodologias de avaliação empregadas, observa-se que em geral avaliações qualitativas são obtidas por meio de observações e entrevistas e as quantitativas são obtidas pela aplicação de pré- e pós- testes.

Muitos objetivos de pesquisa encontrados neste levantamento são semelhantes aos encontrados no Brasil [Bordini et al. 2016b], tais como: atrair a atenção para a área da computação, alguns em especial das meninas; utilizar linguagens de programação visual em experiências de ensino de programação; e buscar formas de avaliar o desenvolvimento de habilidades do PC. Embora muitos trabalhos apresentem metodologias para o ensino de habilidades do PC, muitas são propostas isoladas, o que carece de um modelo conceitual genérico. Igualmente, as propostas de inserção do PC no currículo, seja como disciplina ou interdisciplinarmente, ainda se constituem de propostas independentes e desconectadas, de forma que investigações e fundamentações de quais habilidades do PC e/ou conceitos da computação são adequados a cada idade e/ou ano da educação básica devem ser estabelecidas. Metodologias de ensino para o melhor desenvolvimento de cada uma das dimensões e habilidades do PC também devem ser fundamentadas.

Tabela 1: Artigos Analisados.

\begin{tabular}{|c|c|c|c|c|c|}
\hline ID & Abord. & Ano & Autor & Título & Público \\
\hline 1 & \multirow{17}{*}{ AVAL } & 2012 & Werner et al. & $\begin{array}{l}\text { The Fairy Performance Assessment: Measuring Computational Thinking in middle } \\
\text { school }\end{array}$ & $\mathrm{EF}$ \\
\hline 2 & & 2013 & Bennett et al. & Computing Creativity: Divergence in Computational Thinking & $\mathrm{NE}$ \\
\hline 3 & & 2013 & Gouws et al. & $\begin{array}{l}\text { Computational Thinking in educational activities: an evaluation of the educational } \\
\text { game Light-bot }\end{array}$ & $\mathrm{NE}$ \\
\hline 4 & & 2014 & Moreno et al. & Automatic detection of bad programming habits in scratch: A preliminary study & $\mathrm{NE}$ \\
\hline 5 & & 2014 & Ambrósio et al. & Digital ink for cognitive assessment of computational thinking & $\mathrm{NE}$ \\
\hline 6 & & 2014 & Oliveira et al. & $\begin{array}{l}\text { Quantitative correlation between ability to compute and student Performance in a pri- } \\
\text { mary school }\end{array}$ & $\mathrm{EF}$ \\
\hline 7 & & 2014 & Grover et al. & Assessing Computational Learning in K-12 & EF \\
\hline 8 & & 2015 & Burgett et al. & $\begin{array}{l}\text { DISSECT: Analysis of pedagogical techniques to integrate computational thinking } \\
\text { into K-12 curricula }\end{array}$ & EF, EM \\
\hline 9 & & 2015 & Park et al. & $\begin{array}{l}\text { Effects of gender and abstract thinking factors on adolescents' computer program le- } \\
\text { arning }\end{array}$ & EM \\
\hline 10 & & 2015 & Folk et al. & $\begin{array}{l}\text { GK-12 DISSECT: Incorporating computational thinking with K-12 science without } \\
\text { computer access }\end{array}$ & EF, EM \\
\hline 11 & & 2015 & Seiter et al. & Using SOLO to classify the programming responses of primary grade students & EF \\
\hline 12 & & 2015 & Basawapatna et al. & $\begin{array}{l}\text { Closing the cyberlearning loop: Enabling teachers to formatively assess student pro- } \\
\text { gramming projects }\end{array}$ & EF, EM \\
\hline 13 & & 2015 & Giordano et al. & $\begin{array}{l}\text { New horizons in the assessment of computer science at school and beyond: Levera- } \\
\text { ging on the ViVA Platform }\end{array}$ & EF, EM \\
\hline 14 & & 2015 & Worrell et al. & Collaboration and Computational Thinking: A classroom structure & EF \\
\hline 15 & & 2016 & López et al. & $\begin{array}{l}\text { Visual programming languages integrated across the curriculum in elementary school: } \\
\text { a two year case study using Scratch in five schools }\end{array}$ & EF \\
\hline 16 & & 2016 & Parmar et al. & $\begin{array}{l}\text { Programming moves: design and evaluation of applying embodied interaction in vir- } \\
\text { tual environments to enhance computational thinking in middle school students }\end{array}$ & $\mathrm{EF}$ \\
\hline 17 & & 2016 & Dasgupta et al. & Remixing as a pathway to Computational Thinking & $\mathrm{NE}$ \\
\hline
\end{tabular}


VI Congresso Brasileiro de Informática na Educação (CBIE 2017)

Anais do XXVIII Simpósio Brasileiro de Informática na Educação (SBIE 2017)

Tabela 1 - continuação.

\begin{tabular}{|c|c|c|c|c|c|}
\hline ID & Abord. & Ano & Autor & Título & Público \\
\hline 18 & \multirow{5}{*}{ CONC } & 2012 & Gibson et al. & Teaching graph algorithms to children of all ages & EF, EM \\
\hline 19 & & 2013 & Webb et al. & Using scaffolded examples to teach Computational Thinking concepts & EF \\
\hline 20 & & 2014 & Feaster et al. & Serious Toys: three years of teaching computer science concepts in K-12 Classrooms & EF, EM \\
\hline 21 & & 2016 & Lamprecht et al. & A summer computing camp using ChainReaction and jABC & EM \\
\hline 22 & & 2016 & Mason et al. & Computational Thinking as a liberal study & $\mathrm{NE}$ \\
\hline 23 & \multirow{10}{*}{ CURR } & 2012 & Settle et al. & Infusing Computational Thinking into the middle- and high-school curriculum & EF, EM \\
\hline 24 & & 2012 & Goldberg et al. & Engaging computer science in traditional education: the ECSITE project & EF, EM \\
\hline 25 & & 2013 & Bargury et al. & A nationwide exam as a tool for improving a new curriculum & EF, EM \\
\hline 26 & & 2014 & Cateté et al. & Use and development of entertainment technologies in after school STEM program & EF \\
\hline 27 & & 2014 & Dwyer et al. & $\begin{array}{l}\text { Identifying elementary students' pre-instructional ability to develop algorithms and } \\
\text { step-by-step instructions }\end{array}$ & EF \\
\hline 28 & & 2015 & Franklin et al. & $\begin{array}{l}\text { Getting started in teaching and researching computer science in the elementary class- } \\
\text { room }\end{array}$ & EF \\
\hline 29 & & 2016 & Mouza et al. & $\begin{array}{l}\text { Implementation and outcomes of a three-pronged approach to professional develop- } \\
\text { ment for CS principles }\end{array}$ & EF, EM \\
\hline 30 & & 2016 & Touretzky et al. & Teaching “Lawfulness"with Kodu & $\mathrm{EF}$ \\
\hline 31 & & 2016 & Chiprianov et al. & Introducing Computational Thinking to K-5 in a French context & EF \\
\hline 32 & & 2016 & Tabet et al. & From Alice to Python. Introducing text-based programming in middle schools & $\mathrm{EF}$ \\
\hline 33 & \multirow{5}{*}{ FERR } & 2012 & Ferreira et al. & $\begin{array}{l}\text { Combining cognitive, semiotic and discourse analysis to explore the power of notati- } \\
\text { ons in visual programming }\end{array}$ & $\mathrm{NE}$ \\
\hline 34 & & 2013 & Basawapatna et al. & $\begin{array}{l}\text { The Simulation Creation Toolkit: an initial exploration into making programming } \\
\text { accessible while preserving Computational Thinking }\end{array}$ & EF \\
\hline 35 & & 2014 & Al-Jarrah et al. & "AliCe-ViLlagE"Alice as a collaborative virtual learning environment & EF \\
\hline 36 & & 2014 & Gorman et al. & Learning relational algebra by snapping blocks & EF, EM \\
\hline 37 & & 2016 & Goyal et al. & $\begin{array}{l}\text { Code Bits: an inexpensive tangible Computational Thinking toolkit for K-12 curricu- } \\
\text { lum }\end{array}$ & EF, EM \\
\hline 38 & \multirow{7}{*}{ PROF } & 2013 & Koh et al. & $\begin{array}{l}\text { Will it stick?: exploring the sustainability of Computational Thinking education th- } \\
\text { rough game design }\end{array}$ & $\mathrm{EF}$ \\
\hline 39 & & 2013 & Vieira et al. & $\begin{array}{l}\text { Using backwards design Process for the design and implementation of Computer Sci- } \\
\text { ence (CS) principles: a case study of a Colombian elementary and secondary teacher } \\
\text { development program }\end{array}$ & EF, EM \\
\hline 40 & & 2013 & Bort et al. & $\begin{array}{l}\text { CS4Impact: measuring Computational Thinking concepts present in CS4HS partici- } \\
\text { pant lesson plans }\end{array}$ & EM \\
\hline 41 & & 2014 & Bustillo et al. & Scratching the surface of digital literacy... but we need to go deeper & EF, EM \\
\hline 42 & & 2015 & Israel et al. & $\begin{array}{l}\text { Supporting all learners in school-wide computational thinking: a cross-case qualita- } \\
\text { tive analysis }\end{array}$ & $\mathrm{EF}$ \\
\hline 43 & & 2015 & Byrne et al. & $\begin{array}{l}\text { Empowering teachers to teach CS - Exploring a social constructivist approach for CS } \\
\text { CPD, using the Bridge } 21 \text { model }\end{array}$ & EF \\
\hline 44 & & 2015 & Han et al. & Entry: visual programming to enhance children's Computational Thinking & $\mathrm{EF}$ \\
\hline 45 & \multirow[t]{2}{*}{ JOGO } & 2013 & Werner et al. & $\begin{array}{l}\text { Pair programming for middle school students: does friendship influence academic } \\
\text { outcomes? }\end{array}$ & EF \\
\hline 46 & & 2016 & Horn et al. & $\begin{array}{l}\text { Design insights into the creation and evaluation of a computer science educational } \\
\text { game }\end{array}$ & EF \\
\hline 47 & \multirow{9}{*}{ METO } & 2012 & Webb et al. & Toward an emergent theory of broadening participation in computer science education & EF \\
\hline 48 & & 2013 & Grover et al. & $\begin{array}{l}\text { Using a discourse-intensive pedagogy and Android's App Inventor for introducing } \\
\text { computational concepts to middle school students }\end{array}$ & $\mathrm{EF}$ \\
\hline 49 & & 2014 & Li et al. & Research into the Computational Thinking for the teaching of computer science & $\mathrm{NE}$ \\
\hline 50 & & 2014 & Basawapatna et al. & $\begin{array}{l}\text { The consume - create spectrum: balancing convenience and Computational Thinking } \\
\text { in STEM learning }\end{array}$ & EM \\
\hline 51 & & 2014 & Souza et al. & $\begin{array}{l}\text { Cultural appropriation of Computational Thinking acquisition research: seeding fields } \\
\text { of diversity }\end{array}$ & EF \\
\hline 52 & & 2014 & Daily et al. & $\begin{array}{l}\text { Dancing Alice: exploring embodied pedagogical strategies for learning Computatio- } \\
\text { nal Thinking }\end{array}$ & EF \\
\hline 53 & & 2015 & Li et al. & $\begin{array}{l}\text { Teaching research and practice of blended leaning model based on Computational } \\
\text { Thinking }\end{array}$ & $\mathrm{NE}$ \\
\hline 54 & & 2016 & Razak et al. & Alice in the middle east: an experience report from the formative phase & $\mathrm{EF}$ \\
\hline 55 & & 2016 & Rodriguez et al. & $\begin{array}{l}\text { Using student performance to assess CS Unplugged activities in a classroom environ- } \\
\text { ment }\end{array}$ & EF \\
\hline 56 & \multirow[t]{3}{*}{ DISC } & 2012 & Lewis et al. & $\begin{array}{l}\text { Building upon and enriching grade four mathematics standards with programming } \\
\text { curriculum }\end{array}$ & EF \\
\hline 57 & & 2014 & Arraki et al. & $\begin{array}{l}\text { DISSECT: an experiment in infusing Computational Thinking in K-12 science curri- } \\
\text { cula }\end{array}$ & EF, EM \\
\hline 58 & & 2014 & Basu et al. & Investigating student generated computational models of science & EF \\
\hline
\end{tabular}

Continua na próxima página... 
VI Congresso Brasileiro de Informática na Educação (CBIE 2017)

Anais do XXVIII Simpósio Brasileiro de Informática na Educação (SBIE 2017)

Tabela 1 - continuação.

\begin{tabular}{|c|c|c|c|c|c|}
\hline ID & Abord. & Ano & Autor & Título & Público \\
\hline 59 & \multirow{7}{*}{ DISC } & 2014 & Farris et al. & $\begin{array}{l}\text { Perspectival computational thinking for learning physics: a case study of collaborative } \\
\text { agent-based modeling }\end{array}$ & EF \\
\hline 60 & & 2014 & Towhidnejad et al. & Introducing Computational Thinking through stealth teaching & EF, EM \\
\hline 61 & & 2015 & Peel et al. & $\begin{array}{l}\text { DISSECT: an experiment in infusing Computational Thinking in a sixth grade class- } \\
\text { room }\end{array}$ & $\mathrm{EF}$ \\
\hline 62 & & 2015 & Bean et al. & $\begin{array}{l}\text { Starting from Scratch - developing a pre-service teacher training program in Compu- } \\
\text { tational Thinking }\end{array}$ & EF, EM \\
\hline 63 & & 2015 & Brancaccio et al. & $\begin{array}{l}\text { Problem posing and solving: strategic Italian key action to enhance teaching and lear- } \\
\text { ning mathematics and informatics in the high school }\end{array}$ & EM \\
\hline 64 & & 2015 & Sabitzer et al. & Modeling: a computer science concept for general education & EF \\
\hline 65 & & 2015 & Nesiba et al. & $\begin{array}{l}\text { DISSECT: exploring the relationship between Computational Thinking and English } \\
\text { literature in K-12 curricula }\end{array}$ & EM \\
\hline 66 & \multirow{2}{*}{ PERC } & 2013 & Black et al. & Making computing interesting to school students: teachers' perspectives & EF, EM \\
\hline 67 & & 2014 & Mannila et al. & Computational Thinking in K-9 education & EM \\
\hline 68 & \multirow{6}{*}{ PROG } & 2013 & Kafai et al. & $\begin{array}{l}\text { The social turn in K-12 programming: moving from computational thinking to com- } \\
\text { putational participation }\end{array}$ & EF, EM \\
\hline 69 & & 2014 & Lee et al. & Principles of a debugging-first puzzle game for computing education & EM \\
\hline 70 & & 2015 & Sullivan et al. & CodePlus - designing an after school computing programme for girls & EF, EM \\
\hline 71 & & 2015 & Lakanen et al. & $\begin{array}{l}\text { What does it take to do computer programming? Surveying the K-12 students' con- } \\
\text { ceptions }\end{array}$ & EF, EM \\
\hline 72 & & 2015 & Dorling et al. & Scratch: a way to Logo and Python & EF, EM \\
\hline 73 & & 2016 & Grover et al. & Factors influencing computer science learning in middle school & $\mathrm{EF}$ \\
\hline 74 & \multirow{7}{*}{ ROBO } & 2013 & Touretzky et al. & Using SOLO to classify the programming responses of primary grade students & EF \\
\hline 75 & & 2013 & Larkins et al. & $\begin{array}{l}\text { Application of the cognitive apprenticeship framework to a middle school robotics } \\
\text { camp }\end{array}$ & $\mathrm{EF}$ \\
\hline 76 & & 2013 & Cross et al. & A visual robot-programming environment for multidisciplinary education & EF \\
\hline 77 & & 2015 & Sanneman et al. & A distributed robot garden system & $\mathrm{NE}$ \\
\hline 78 & & 2015 & Rode et al. & From Computational Thinking to computational making & $\mathrm{EF}$ \\
\hline 79 & & 2016 & Atmatzidou et al. & $\begin{array}{l}\text { Advancing students' computational thinking skills through educational robotics: a } \\
\text { study on age and gender relevant differences }\end{array}$ & EM \\
\hline 80 & & 2016 & Eguchi et al. & $\begin{array}{l}\text { RoboCupJunior for promoting STEM education, } 21 \text { st century skills, and technological } \\
\text { advancement through robotics competition }\end{array}$ & EM \\
\hline
\end{tabular}

\section{Referências}

Andrade, D. et al. (2013). Proposta de atividades para o desenvolvimento do pensamento computacional no ensino fundamental. In Anais do WIE 2013, pages 169-178.

Bell, T., Witten, I. H., and Fellows, M. (1998). Computer Science Unplugged: Off-line activities and games for all ages.

Bordini, A. et al. (2016a). Computação na educação básica no Brasil: o estado da arte. Revista de Informática Teórica e Aplicada, 23(2):210-238.

Bordini, A. et al. (2016b). Desdobramentos do pensamento computacional no Brasil. In Anais do SBIE 2016, pages 200-209.

Martins, L. et al. (2016). Ensinando lógica de programação aplicada a robótica para alunos do ensino fundamental. In Anais do SBIE 2016, pages 31-41.

Mota, F. et al. (2014). Desenvolvendo o raciocínio lógico no ensino médio: uma proposta utilizando a ferramenta scratch. In Anais do SBIE 2014, pages 377-381.

Oliveira, E. et al. (2016). Pensamento computacional e robótica: Um estudo sobre habilidades desenvolvidas em oficinas de robótica educacional. In Anais do SBIE 2016, pages 530-539.

Togelius, J. et al. (2011). Search-based procedural content generation: A taxonomy and survey. IEEE Transact. on Computational Intelligence and AI in Games, 3:172-186.

Wing, J. M. (2006). Computational thinking. Communications of the ACM, 49(3):33-35. 